\title{
Archives
}

\section{L'influence du Service des archives de l'Université de Montréal sur la pratique archivistique au Québec : une affaire de fierté (Témoignage)}

\section{DENYS CHOUINARD}

Volume 46, numéro 2, 2017

$50^{\mathrm{e}}$ anniversaire de la DGDA

URI : https://id.erudit.org/iderudit/1040384ar

DOI : https://doi.org/10.7202/1040384ar

Aller au sommaire du numéro

Éditeur(s)

Association des archivistes du Québec (AAQ)

ISSN

0044-9423 (imprimé)

2369-9256 (numérique)

Découvrir la revue

Citer ce document

Chouinard, D. (2017). L'influence du Service des archives de l'Université de Montréal sur la pratique archivistique au Québec : une affaire de fierté (Témoignage). Archives, 46(2), 119-122. https://doi.org/10.7202/1040384ar
Résumé de l'article

Denys Chouinard utilise le prétexte du $50^{\mathrm{e}}$ anniversaire de la DGDA de l’Université de Montréal pour souligner le caractère novateur et la contribution exceptionnelle de ce service à l'archivistique. Dès les années 1960 et 1970 le Service des archives de l'Université de Montréal s'est démarqué et ce caractère avant-gardiste s'est confirmé dans les années 1980. Il rappelle le plaisir qu'il eut à travailler auprès de Jacques Ducharme, inspirant par son enthousiasme et la façon positive qu'il avait d'exercer sa profession. L'exercice de gestion par objectif mis en place au service des archives eut un effet dynamisant sur l'équipe en place et le service est toujours resté à l'affût des opportunités d'augmenter ses ressources, tant humaines que financières, afin d'atteindre les objectifs fixés. Plusieurs autres services d'archives s'en sont inspirés. L'auteur nous présente ensuite une liste de réalisations importantes du service des archives de l'UdeM et mentionne au passage l'influence qu'ont eu ses années passées au sein du service sur l'ensemble de sa carrière. Il termine en évoquant l'approche axée sur les utilisateurs telle que pratiquée à la DGDA et souligne qu'elle fait maintenant partie de la Déclaration universelle sur les archives. Pour Denys Chouinard, le Service des archives de l'Université de Montréal a raison d'être fier d'avoir une telle influence. 


\section{ANNIVERSAIRE DE LA DGDA}

\section{L'influence du Service des archives de l'Université de Montréal sur la pratique archivistique au Québec: une affaire de fierté (Témoignage)}

\section{DENYS CHOUINARD}

Archiviste

Ceux qui me connaissent savent que je n'ai qu'une façon de me présenter, la plus simple et la plus claire: «Je m'appelle Denys Chouinard, je suis archiviste ». Dans les minutes qui suivent, vous comprendrez d'où vient cette manière de faire. Ce sera en quelque sorte ma part à la fête qui nous rassemble.

Le Service des archives de I'Université de Montréal (UdeM) a contribué autant à la discipline qu'à la profession archivistique. Dans les années 1960 et 1970, la combinaison exceptionnelle des visions et des talents de certaines personnes a donné à l'Université de Montréal le début canon d'un leadership sans égal depuis. Même les grandes institutions nationales en archivistique n'ont pas eu un tel impact. Les archivistes de I'UdeM, et je pèse mes mots, ont joué en quelque sorte le rôle de bougie d'allumage d'une Révolution tranquille de la gestion de documents et des archives. 
Débordement d'enthousiasme de ma part? Pas vraiment. Les années que j'ai vécues à l'Université de 1981 à 1990 furent parmi celles où ses archivistes proposaient à tout le milieu archivistique, petits et grands services d'archives, une nouvelle approche de la discipline. Aujourd'hui, que devons-nous aux archivistes de l'UdeM? Quelles sont leurs réalisations propres, celles qu'ils ont pilotées ou auxquelles ils ont contribué et qui ont marqué notre société?

Ce fut au-delà de toute attente. Loin de moi l'ambition de tout énumérer. À vouloir être exhaustif, on oublie forcément des activités et des gens. Je m'en tiendrai à l'essentiel. Je veux mentionner ici comment I'Université a été servie en matière de gestion de ses propres documents administratifs et de son patrimoine archivistique et souligner ensuite de quelle façon on a créé du savoir - de l'expertise - au profit de la collectivité, comme doivent le faire tous les établissements universitaires.

Mais dans le temps qui m'est accordé, je fais le choix de vous entretenir non pas tant de ce savoir-faire que du savoir-être archivistique issu de la pratique de la profession à l'Université de Montréal. Chaque fois que je circule dans le pavillon Roger-Gaudry, je me rappelle le regretté collègue Jacques Ducharme. J'ai encore frais à la mémoire la présentation plus qu'enthousiaste qu'il donnait de ses projets; je le revois démontrer une énergie hors du commun et tellement communicative. À son contact, nous nous sentions transporté. Tout semblait possible; il était exactement à l'opposé de l'archiviste misérabiliste se plaignant de ne pas être reconnu par la société. Il avait la passion du document, de l'organisation du travail, de la réalisation des objectifs fixés. II n'avait résolument aucun temps pour le dénigrement, pour quelque attitude négative que ce soit.

La pratique de l'archivistique implique toute une série de mesures pour assurer la bonne circulation et la gestion des documents. Matériellement, les boîtes, les magasins d'archives avec leurs rayonnages deviennent la face visible du travail de l'archiviste. Mais pour celles et ceux qui s'en tiennent à la technique de cette gestion, qui se soucient beaucoup plus du contenant que du contenu, en d'autres mots qui ne connaissent pas I'histoire de leur institution et de ses unités révélée par les documents, le risque est grand d'être perçu comme de simples gestionnaires d'entrepôts. Et c'est là que l'archiviste n'est plus un professionnel, mais strictement un exécutant. Jacques Ducharme était à des années-lumière de cette image.

Je me rappelle très bien qu'à mon entrée au Service en 1981, on m'a immédiatement initié à la formule du travail par objectifs. À raison de 
deux réunions par année, les trois cadres du Service, après avoir rencontré les membres de leurs équipes respectives, faisaient le point sur le travail réalisé au cours du semestre passé et établissaient les attentes pour les six mois à venir. II y avait là un exercice éminemment profitable de contrôle et d'évaluation pour se faire une excellente idée du rendement; c'était ensuite une façon optimale de planifier l'avenir immédiat, de dynamiser les troupes pour que chaque membre de l'équipe sache très clairement où nous allions et veuille apporter sa contribution. Afin de conserver une mémoire précise de la progression de l'unité, le rapport annuel permettait de consigner les informations indispensables sur les ressources utilisées et les activités réalisées. Chacune et chacun pouvait ainsi se rappeler la direction donnée et vérifier les résultats obtenus. La formule n'en est pas une de contrôle tatillon, mais bien un puissant stimulant pour motiver les troupes. Combien profitable elle a été.

Mais il va de soi aussi que ce mode de fonctionnement se bute très vite au manque possible de ressources humaines et financières pour progresser au rythme ambitieux souhaité. C'est ce qui a amené le Service à ouvrir très tôt ses portes aux stagiaires et à multiplier les demandes de subventions. Cet apport de nouvelles ressources engendrant un accroissement de travail n'a jamais été perçu négativement. Au contraire, nous y avons vu une chance de progresser plus rapidement, d'atteindre plus facilement les objectifs visés. Ce mode de fonctionnement, toujours en vigueur ici, a été très largement repris dans tout le milieu archivistique québécois.

Faute de temps, laissez-moi évoquer en rafale des réalisations marquantes du Service: début de l'utilisation de l'informatique dès les années 1970 pour la constitution des instruments de recherche, publication de ces derniers, participation à des émissions de radio et de télévision pour faire connaître les archives de l'Université; en somme, des membres d'un service $d^{\prime}$ archives constamment aux aguets pour donner un sens à leur travail, c'est-à-dire communiquer le patrimoine acquis, traité et conservé. II n'y avait aucune raison à tout cela si ça n'aboutissait pas à la diffusion du patrimoine archivistique. Ainsi, riche d'un savoir-faire, il n'était pas question de le garder pour soi. Nous nous faisions I'obligation de le partager sous forme de conférences, de cours, de participation aux congrès de l'AAQ, de publications d'articles de revues scientifiques et de manuels. Nous ne voulions pas être spectateurs, mais plutôt des joueurs qui constituent le premier trio sur la glace.

J'ai passé les dix premières années de ma carrière archivistique à I'Université de Montréal. Les dix-huit suivantes à la Ville de Montréal et les 
cinq dernières à la Congrégation de Notre-Dame. Tout ce que j'ai pu réaliser avec les équipes motivées et talentueuses à la Ville et à la Congrégation vient directement de mon Alma Mater, le Service des archives de I'Université de Montréal. Agir avec dynamisme en matière d'acquisition, obtenir le maximum de subventions gouvernementales pour accélérer le traitement des archives, en obtenir tout autant sinon plus pour multiplier la production d'expositions réelles ou virtuelles, réunir les conditions matérielles pour assurer la conservation permanente des documents, tout cela découle de mes premiers apprentissages à l'Université. D'autres dans le milieu archivistique québécois ont fait leur cette philosophie.

Cette approche, tournée vers les utilisateurs des archives et non pas platement et bêtement corporatiste, a été inscrite directement dans la Déclaration québécoise sur les archives devenue Déclaration universelle endossée par I'UNESCO en 2011; je tiens à noter que c'est avec la directrice actuelle du Service des archives de l'Université, mon amie Diane Baillargeon, que j'ai eu la chance de piloter ce dossier.

En conclusion, je dirai que lorsque j'évoque ainsi l'influence du Service des archives de l'Université de Montréal sur la pratique archivistique au Québec, c'est d'une histoire de fierté dont je parle. La fierté ! Ce sentiment qui fait sauter les blocages, qui encourage les initiatives, qui pousse les individus bien au-delà de ce qu'ils pensent être en mesure de réaliser.

J'ai fait partie de cette école, le mot est juste, cette école qu'est le Service des archives de l'Université de Montréal. Aujourd'hui, je suis heureux d'avoir momentanément interrompu ma retraite et d'être venu partager ce bonheur avec vous. Je retourne maintenant sur mes terres, l'esprit toujours habité par les riches souvenirs d'une expérience humaine qui m'a comblé parce qu'elle faisait grandir. J'ose croire qu'elle a influencé des générations d'archivistes qui ont pu ainsi mieux servir leurs institutions en leur permettant de s'approprier leur patrimoine et de l'intégrer dans leur actualité. En échange, ces archivistes ont reçu tout comme moi la reconnaissance et l'estime de leurs pairs et de leurs concitoyens à titre de professionnels. Ça n'a pas de prix!

Aux membres du Service des archives de l'Université de Montréal d'aujourd'hui, je leur dis bon anniversaire, bravo d'avoir eu la sagesse de jeter un coup d'œil derrière vous par le présent colloque, et bonne route dans la poursuite de la mise en valeur du trésor dont vous êtes gardiens. Puissiez-vous continuer de prôner la meilleure définition des archives, celle qui n'a rien de nostalgique, rien de figé dans le passé. Celle qui dit tout simplement que les archives, c'est la vie! 